\title{
Insulin-like Growth Factor-I Governs Submucosal Growth and Thickness in the Newborn Mouse Ileum
}

\author{
ANDREW C. HERMAN, ERICA M. CARLISLE, JESSICA B. PAXTON, AND PHILLIP V. GORDON \\ Division of Neonatology, Department of Pediatrics, University of Virginia Health System, Charlottesville, \\ Virginia 22908, U.S.A.
}

\begin{abstract}
ABSTR
Spontaneous intestinal perforations in extremely premature
infants are associated with glucocorticoid-induced thinning of
the ileal bowel wall. We have previously demonstrated that
insulin-like growth factor-1 (IGF-1) is abundant within the sub-
mucosa of the newborn mouse ileum but is diminished by
glucocorticoid exposure, concomitant with bowel wall thinning.
These findings prompted us to hypothesize that IGF-I governs
submucosal growth during neonatal gut development and that
diminished IGF-I abundance results in submucosal thinning.
Heterozygous IGF-I knockout, wild type and homozygous IGF-I
over-expresser newborn mice were euthanized at 3 d of life.
Additionally, wild type newborn mice received daily dexameth-
asone (DEX) ( $1 \mu \mathrm{g} / \mathrm{gm} / \mathrm{d}$ ) or vehicle control on days of life 1 and
2 and were also euthanized at 3 d of life. Their ileums were
harvested, fixed and the resulting sections were processed in
parallel for IGF-I immunohistochemistry and morphometric
measurements of submucosal thickness and bowel diameter.
Immunolocalization of IGF-I in each genotype was also com-
pared with that seen in DEX-treated and control mice euthanized
\end{abstract}
ABSTRACT

at the same time of life. IGF-I heterozygous knockouts had diminished submucosal IGF-I immunolocalization (similar to that seen in DEX-treated newborn mice) whereas homozygous IGF-I over-expressers exceeded that seen within wild type mice. IGF-I genotype and submucosal abundance was positively correlated with ileal submucosal thickness. DEX treatment of newborn mice diminished IGF-I and caused significant thinning of the submucosa compared with controls. Submucosal growth and thickness in the neonatal mouse ileum is governed by IGF-I and is diminished by dexamethasone treatment. (Pediatr Res 55: 507-513, 2004)
DEX, dexamethasone
OE, over-expressor
KO, knockout
WT, wild type
IGF-1, insulin-like growth factor-1

Abbreviations
Focal or spontaneous intestinal perforation in the extremely low birth weight infant is an emerging disease that was not commonly seen before the introduction of surfactant therapy. Today, now that the age of gestational viability has been significantly lowered, $4 \%$ to $5 \%$ of infants born weighing less than $1000 \mathrm{~g}$ will develop a sterile ileal perforation in the first week of life and twice that many will perforate if exposed to higher doses of glucocorticoids in the perinatal period (1). This disease causes significant morbidity and mortality in the neonate due to the subsequent development of peritonitis. Spontaneous perforations also represent a disturbing conundrum in neonatology, i.e. that effective therapies for prevalent develop-

Received April 28, 2003; accepted October 15, 2003

Correspondence: Phillip V. Gordon M.D., Ph.D., Division of Neonatology Department of Pediatrics, PO Box 800386, University of Virginia Health System, Charlottesville, VA 22908; e-mail: pvg4n@virginia.edu

Support for this project was provided through grants from the NIH: CHRCDA HD01421 (P.V.G) and NIDDK 1KO8DK/HD61553-01 (P.V.G.).

DOI: 10.1203/01.PDR.0000110525.30786.50 mental diseases (like respiratory distress syndrome) will likely result in the emergence of new and equally morbid diseases in the rescued survivors. In some cases, preexisting adjunct therapies can actually facilitate the genesis of such diseases.

For example, several prominent clinical studies have demonstrated a relationship between perinatal dexamethasone treatment and spontaneous intestinal perforations (2-4). Histologic studies of perforated ileal tissue demonstrate a skewing of tissue development such that the mucosa is hypertrophied, the lumen is dilated and the bowel wall is thinned (2). These findings are consistent with a steroid-induced perturbation, since glucocorticoids are known to mediate mucosal maturation during weaning and to induce catabolism within the mesenchymal tissues of the intestine during prolonged exposure (5-7). These events suggest that the combination of lumen dilation and bowel wall thinning creates a vulnerability to perforation within the immature ileum of the extremely low birth weight infant $(2,8)$.

We have previously used a newborn mouse model (where dexamethasone treatment recapitulates the histologic findings 
in extremely low birth weight infants) to better understand the mechanisms by which steroids perturb development in the neonatal ileum (9). Results from these experiments suggest that IGF-I is an important mediator of glucocorticoid effect in the neonatal ileum. In brief, dexamethasone treatment was associated with a redistribution of IGF-I from the submucosa and lamina propria to epithelial cells within the distal villus, paralleling the observed trophic changes associated with perforation (2).

In this study, we compared the submucosal thickness of neonatal mouse ileums in heterozygous IGF-I knockout (because homozygous progeny have poor viability), wild type, and homozygous IGF-I over-expressing transgenic mice to examine the relationships between IGF-I abundance and submucosal thickness. Parallel measurements were also performed in dexamethasone-treated and vehicle-control treated mice. Our goal was to test the hypothesis that submucosal growth and thickness is dependent on IGF-I availability in the neonatal mouse. Our findings support the supposition that steroid-induced paucity of submucosal IGF-I results in bowel wall thinning within the neonatal ileum.

\section{MATERIALS AND METHODS}

Mice. Animal protocols used for this study were approved by the Institutional Animal Care and Use Committee at the University of Virginia. Four mice from each transgenic condition and 8 dexamethasone-treated and 8 vehicle-control mice were used for morphologic measurement and immunohistochemistry. Transgenic mice were weighed on the day of birth and daily thereafter until euthanasia.

Dexamethasone treatment paradigm. Newborn littermate, wild type mice received dexamethasone $(1 \mu \mathrm{g} / \mathrm{gm} / \mathrm{d})$ or vehicle control by intraperitoneal injection on days of life one and two and then were euthanized on the third day of life (in parallel with the euthanasia times for IGF-I knockout and IGF-I overexpresser mice). This dose of dexamethasone approximates that used in one of the early clinical trials of prophylactic dexamethasone to prevent chronic lung disease (which resulted in a $17 \%$ perforation rate within treated extremely low birth weight infants and prompted the human safety committee to intervene) (3). The chosen treatment period $(72 \mathrm{~h})$ is the earliest time at which optimal IGF-I depletion within the submucosa is evident within dexamethasone-treated newborn mice (unpublished data). Terminal ileums were dissected and processed for immunohistochemistry and morphometric analysis.

IGF-I heterozygous knockout mice. Heterozygous female IGF-I knockout mice were obtained from Dr. Argiris Efstratiadis (Department of Genetics and Development, Columbia University, NY, NY) and bred with agouti wild type males to generate heterozygous IGF-I knockout newborns for this study (Fig. 2A) (10). The heterozygous animals were selected by weight phenotype, euthanized at $72 \mathrm{~h}$ of life and their ileums harvested for immunohistochemistry and morphometric analysis. Heterozygous progeny were used because the homozygous knockout condition is commonly lethal in the newborn
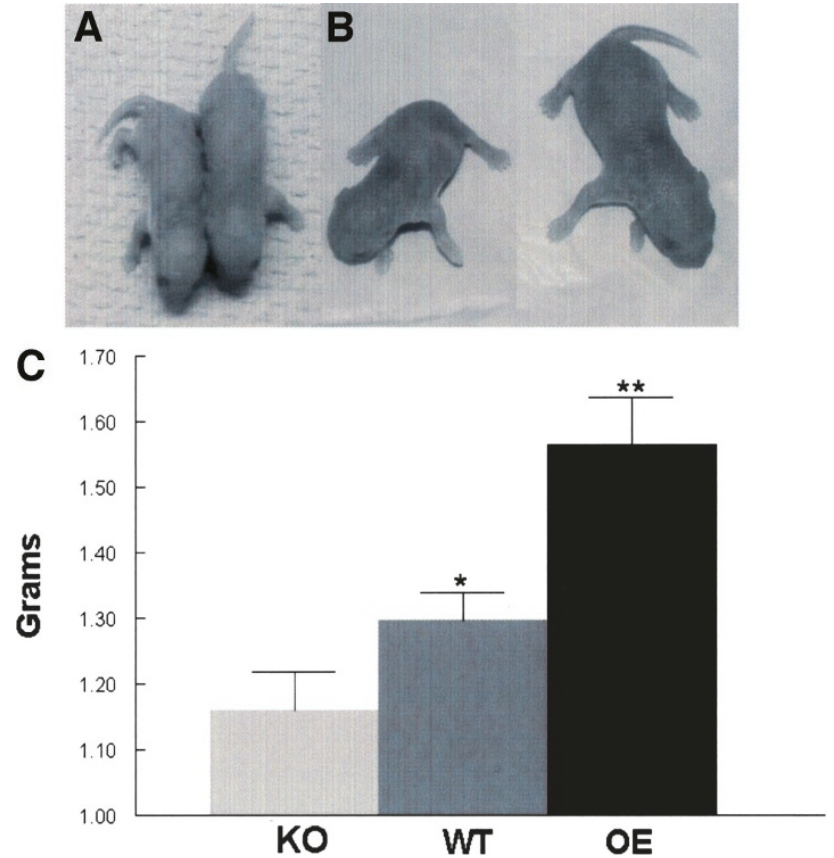

Figure 1. Weight phenotypes for heterozygous IGF-I knockout (KO), wild type (WT) and homozygous IGF-I over-expressing mice (OE). Digital photographs were taken of representative littermate mice on the day of euthanasia. (A) Littermates from an IGF-I heterozygous $\mathrm{KO}$ mother and WT father showing a heterozygous IGF-I KO on the left and a WT on the right. $(B)$ Littermates from IGF-I hemizygous parents showing a WT on the left and an IGF-I homozygous OE on the right. $(C)$ Mean weights at time of euthanasia for all offspring with the selected genotypes $(*=p<0.05$ versus $\mathrm{WT}$; $* *=p<$ 0.05 versus both $\mathrm{KO}$ and WT; error bars $=\mathrm{SD}$; and $n=7 \mathrm{KOs}, 11 \mathrm{WTs}$ and $6 \mathrm{OEs})$.

period and survivors tend to be stressed and less well nourished (making them unsuitable for our experimental paradigm).

IGF-I homozygous over-expresser mice. Hemizygous female human-IGF-I over-expresser mice, with expression driven by the metallothionein promoter, were obtained from Dr. Joe D'Ercole (courtesy of Dr. Kay Lund's colony, University of North Carolina, Department of Pediatrics, Division of Endocrinology, Chapel Hill, NC) and bred with agouti wild type males to generate hemizygous males (see Fig. 2B for breeding and phenotype strategy) $(11,12)$. The genotype of the second generation was confirmed by comparison with first generation hemizygous females (as positive controls) and known wild types (as negative controls) using PCR detection of the human IGF-I transgene with the following primers and parameters:

$5^{\prime}$ oligo $=5^{\prime}$-GGACCGGAGACGCTCTGCGG 3'

$3^{\prime}$ oligo $=5^{\prime}$ GCGGTGGCATGTCACTCTTC 3'

1st cycle: $95^{\circ}$ for $45 \mathrm{~s} ; 55^{\circ}$ for $2 \mathrm{~min} ; 72^{\circ}$ for $5 \mathrm{~min}$.

Cycles 2-31: $95^{\circ}$ for $30 \mathrm{~s} ; 55^{\circ}$ for $90 \mathrm{~s} ; 72^{\circ}$ for $2 \mathrm{~min}$.

The P-53 gene was used as a control, confirming equivalent RNA loading between lanes and conditions.

Males from the second generation were bred with first generation females to yield homozygous human IGF-I overexpresser newborns for this study (Fig. 2B). These animals were selected by weight phenotype as previously described (13), euthanized at $3 \mathrm{~d}$ of life and their ileums were harvested for immunohistochemistry and morphometric analysis. 
$\underline{\mathbf{A}}$
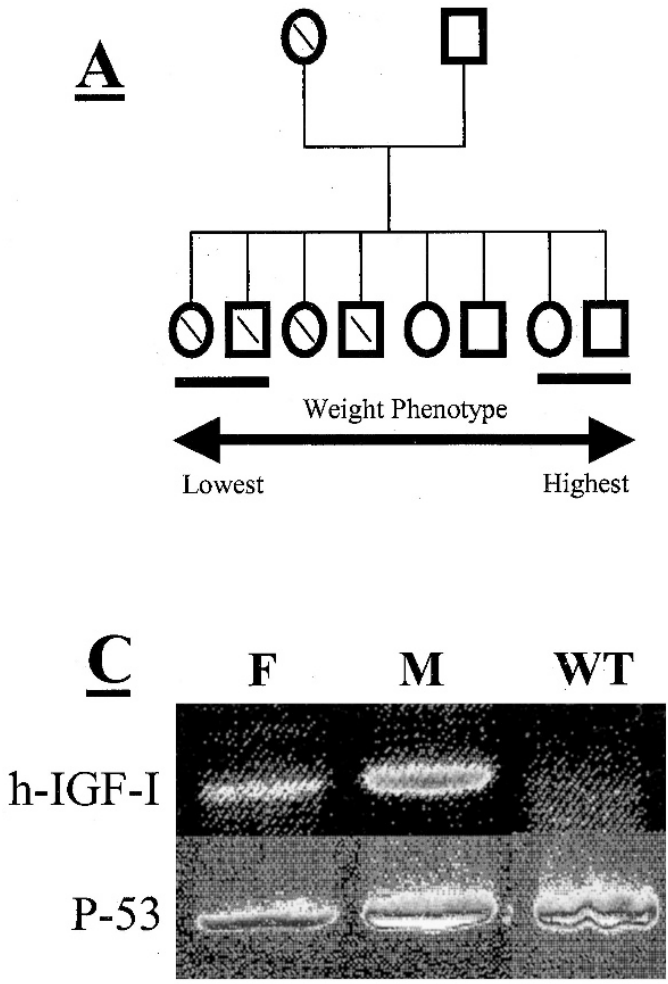

$\underline{\mathrm{D}-\boldsymbol{i}}$

\section{IGF-I KO WT OE}

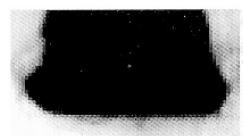

$\underline{\mathbf{B}}$
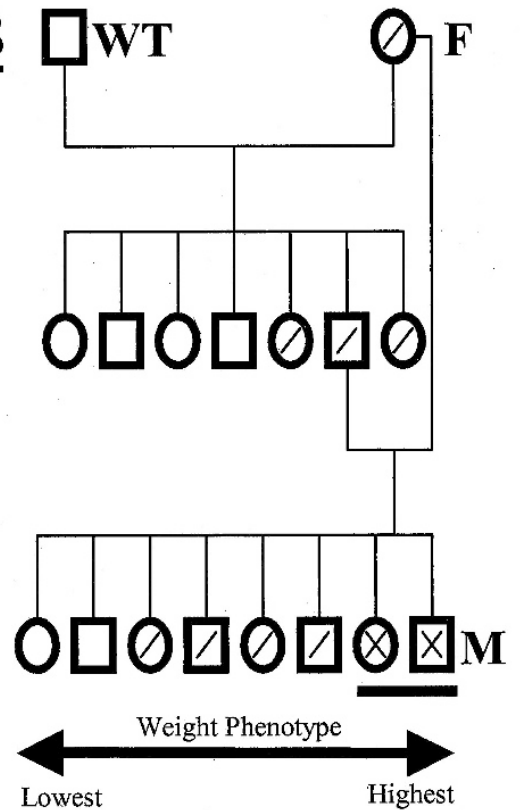

Figure 2. Breeding scheme and genotype confirmation for heterozygous IGF-I knockout (KO), wild type (WT) and homozygous over-expresser (OE) mice. ( $A$ ) Representative pedigree demonstrating the breeding strategy for generation of IGF-I heterozygous KO (marked with back slashes; circles = females; boxes = males) and WT mice (empty circles and boxes). Mice selected for this study by euthanasia weight phenotype are indicated by an underline. (B) Representative pedigree demonstrating the breeding strategy for generation of IGF-I homozygous OE mice (marked with an " $x$ "). Hemizygous IGF-I OEs are indicated by a forward slash and homozygous OE mice selected for this study by euthanasia weight are indicated by an underline. (C) Second generation males used for breeding to homozygosity (M) were compared with first generation females (F) and wild types (WT) by PCR analysis to confirm the presence of the human transgene (h-IGF-I) (their pedigree position is indicated by the same letters in Fig. $2 B$ ). The P-53 gene was used as a control. $(D)$ Western blot quantification of IGF-I in serum, harvested at the time of euthanasia (Di). IGF-I protein standard is shown on the left, with KO, WT and OE showing differing abundance with each genotype. Ponceau S staining of these same samples reveals comparable amounts of loaded protein for each of the three transgenic conditions (Dii).

Wild type mice. Agouti wild type males were bred with IGF-I heterozygous knockout females resulting in $50 \%$ wild type and 50\% IGF-I heterozygous progeny (Fig. 2A). Wild types were discerned from IGF-I knockouts by weight phenotype, euthanized at $3 \mathrm{~d}$ of life and their ileums harvested for immunohistochemistry and morphometric analysis.

Weight phenotype prediction of newborn mouse IGF-I genotype. Heterozygous IGF-I knockout, wild type and IGF-I homozygous over-expressers were selected by weight phenotype (examples shown in Fig. $1 A$ and $B$ ). To determine whether phenotypic selections within individual litters would reflect true genotypic differences across litters (which might be confounded by litter-specific variances in nurture, timing of weighing or background), weights at euthanasia for each IGF-I category were averaged and compared by analysis of variance. The four mice with euthanasia weights closest to the cohort mean for each of the three phenotypes were then selected for further analysis and genotype confirmation.

Western blot confirmation of genotype using newborn mouse serum. Western blot analysis was performed to confirm differences in serum IGF-I abundance across genotypes. Whole blood was isolated from euthanized newborn mice after decapitation by catching cervical artery blood in a centrifuge tube. These samples were centrifuged for $10 \mathrm{~min}$ at 13,000 revolutions per minute two consecutive times and $2.5 \mu \mathrm{L}$ of each resulting supernatant was used for $20 \%$ PAGE, as described by 
Laemmli (14). IGF-I mouse antibody (originally created by Dr. Judd Van Wyk) was obtained from the Developmental Studies Hybridoma Bank - developed under auspices of the NICHD (product \# sm1.2, University of Iowa, Department of Biologic Sciences, IA City, IA, U.S.A.) and used for immunoblotting. Secondary antibody, biotin-avidin amplification and alkaline phosphatase visualization of IGF-I was performed using a commercially available kit from R\&D Systems (Minneapolis, MN, U.S.A.). Ponceau S staining was performed on each blot to demonstrate similarity in protein load across lanes and samples.

Immunohistochemistry. Terminal ileum was fixed in 10\% buffered formalin, cryoprotected in a 30\% sucrose gradient overnight, as previously described by Keller et al. (15), and cut into $5 \mu \mathrm{m}$ sections by cryostat. Alternatively, terminal ileum from dexamethasone-treated and control mice was fixed in $10 \%$ buffered formalin, embedded in paraffin and sectioned into $5 \mu \mathrm{m}$ sections by microtome. Sections from each IGF-I genotype were paired on the same slide, such that IGF-I knockouts, wild types and IGF-I over-expressers were processed in parallel for IGF-I immunohistochemistry to allow direct comparison. Likewise, dexamethasone-treated and vehicle-control ileal sections were paired on the same slide, deparaffinized with xylene and re-hydrated using decreasing ethanol concentrations to a final solution of distilled water.

Immunohistochemistry was performed for IGF-I as previously described (9). Briefly, endogenous peroxidase activity was quenched in $3 \% \mathrm{H}_{2} \mathrm{O}_{2}$ and $60 \%$ methanol for $15 \mathrm{~min}$., followed by 3 washes of distilled water and application of blocking solution $(1 \% \mathrm{wt} / \mathrm{vol} \mathrm{BSA}$ in TBS [0.05 M Tris-HCl, $0.138 \mathrm{M} \mathrm{NaCl}, 0.0027 \mathrm{M} \mathrm{KCl}, \mathrm{pH} 8.0]$ ) for $1 \mathrm{~h}$ at room temperature. This was followed by a one-hour incubation with mouse anti-IGF-I primary antibody (Developmental Studies Hybridoma Bank, IA City, IA, U.S.A.). Primary antibody was washed off with TBS $\times 3$ and secondary antibody (biotinylated anti-mouse antibody [Vector Labs, Burlingame, CA, U.S.A.]) was placed on the slides for $1 \mathrm{~h}$. ABC-HRP (Vector Labs) was prepared and applied per the manufacturer's instructions, washed as above and visualized with peroxidase substrate solution (Vector Labs). The reaction was terminated after 5 min by aspiration of the substrate solution and washing in distilled water for $10 \mathrm{~min}$. at room temperature. The sections were counterstained with hematoxylin and cover-slipped.

Digital photomicroscopy. Digital images of photomicrographs were obtained using a PixCell II laser dissection microscope (Arcturus Engineering, Mountain View CA, U.S.A.). These images were taken in parallel with a micrometer image using the same magnification and saved in Adobe Photoshop (Adobe Systems Inc., San Jose, CA. U.S.A.). The resulting images were laid out in sequence within Adobe Illustrator (Adobe Systems Inc.) and printed along with the micrometer for the purpose of morphologic measurements.

Morphologic measurements: submucosal thickness. Submucosal thickness was measured using 20X micrographs encompassing approximately one fourth of the tissue in each cross section. Measurements were made between the lowest basal margin of epithelial cells within the invaginations of the mucosa (these represent the emerging epithelial cell zones of proliferation at this point in development) and the highest myocytes within the circumferential smooth muscle. These measurements were taken at every mucosal invagination within the digital image and averaged for each transgenic and treatment condition to provide a quantitative comparison of submucosal thickness.

Lumen circumference. Because we have previously shown that cross section circumference is negatively correlated with tissue thickness in the bowel wall, we estimated the lumen circumference using previously described methodology to assure that there was no confounding effect upon submucosal thickness (9). In brief, $10 \times$ digital photomicrographs of ileal cross sections stained with hematoxylin were used to calculate the approximate lumen circumference in the following manner. First, these images were printed in parallel with an image of a micrometer taken from the same lens. Secondly, a line was drawn such that it bisected the lumen equally and was the maximal possible distance from the inner aspect of the circumferential muscularis layer on one side to the same point on the opposite side (thereby drawing it in parallel with any possible skewing of the tissue during sectioning). This first line was then bisected by a second line at a $90^{\circ}$ angle, which also was drawn to the closest inner edge of the muscularis. The second line was then used as the radius, with the distance measured according to the units of the micrometer, since it traverses the distance from the exact middle of the first line to the edge of the muscularis. This value was then multiplied by 2 times pi to calculate the circumference. The resulting values were averaged for each genotype to investigate potential differences and possible confounding effects upon the submucosal thickness.

Muscularis thickness. Muscularis thickness in transgenic animals was measured using 20X photomicrographs encompassing approximately one fourth of the tissue in each cross section. Measurements across the muscularis were made at the point beneath each invagination of the mucosa (using a printed digital image as outlined during methods for measuring submucosal thickness) to provide exact parity between submucosal and muscularis thickness measurements. The values were averaged within each transgenic condition, thereby providing a quantitative comparison of muscularis thickness.

Statistics. Means and standard deviations were calculated for each of the measurements used to compare and contrast the three transgenic conditions. Comparisons between these conditions were made using one-way analysis of variance tests, such that each condition was tested for significant difference between each of the transgenic conditions using SigmaStat software (SPSS, Chicago, IL, U.S.A.). Comparisons between dexamethasone-treated and control animals were assessed by a student $t$ test. Significance was defined as $p \leq 0.05$.

\section{RESULTS}

Weights at time of euthanasia are significantly different between wild types and transgenic mice with contrasting IGF-I genotypes. IGF-I heterozygous knockouts and IGF-I homozygous over-expressers had significantly different weights at time of euthanasia from wild types and were also significantly different from each other, supporting the suppo- 
sition of real differences associated with genotype (Fig. 1). Mean weight at euthanasia for knockouts $(n=7)$ was $1.16 \pm$ $0.06 \mathrm{~g}$, wild types $(n=11)$ was $1.30 \pm 0.04 \mathrm{~g}$ and overexpressers $(n=6)$ was $1.57 \pm 0.07 \mathrm{~g}$. These results suggest that weight may be a reliable means to assess IGF phenotype in the neonatal mouse.

Confirmation of the human IGF-I transgene in 2nd generation over-expresser mice and breeding of homozygous progeny. Although phenotype selection by weight has been reported to be reliable with both transgenic strains used in this study, we found it more difficult to distinguish wild types from hemizygous IGF-I over-expresser mice during the newborn period (13). Because we had planned to breed to homozygosity, the genotypes of hemizygous 2nd generation males were verified by PCR (Fig. 2C). There is a greater h-IGF-I DNA abundance in the hemizygous mice than in controls while the relative equality in DNA loading concentrations is demonstrated by P-53 controls below. This strategy allowed 1st generation - 2nd generation pairings and yielded homozygous phenotypes in a fourth of the resulting progeny.

Serum IGF-I protein abundance correlates with IGF-I gene dosage. Western blot analysis demonstrates that serum IGF-I protein increased with increasing IGF-I gene abundance. Comparing serum samples at the time of euthanasia, IGF-I was undetectable in KO's, minimally detected in wild types and reliably detected in OE's (Fig. 2Di). Ponceau S staining displayed comparable protein load across lanes and samples as a control (Fig. 2Dii). We note that the shape of the band of IGF-I was consistently warped to a "barbell" like configuration, which was likely due to over-riding plasma proteins.

IGF-I abundance is decreased in the ileal submucosa by dexamethasone treatment in newborn mice. IGF-I immunohistochemistry of the three day-old mouse ileums showed a qualitative reduction in labeling within the submucosa of dexamethasone-treated subjects when compared with littermate controls (Fig. $3 A$ and $B$ ). However, staining of IGF-I within the epithelial cell cytoplasm was increased in the dexamethasonetreated ileal sections when compared with controls (not shown).

Submucosal thickness is decreased in dexamethasonetreated mice. Measurements of submucosal thickness from dexamethasone-treated mouse ileums were significantly thinner than those of littermate controls $(p<0.01)$ (Fig. 3C). The mean ileal submucosal thickness and standard deviations from dexamethasone-treated mice $(n=8$ mice and 34 measurements) was $6.49 \pm 3.57 \mu \mathrm{m}$ with a mean submucosal thickness of $16.7 \pm 5.64 \mu \mathrm{m}$ in controls $(n=8$ mice and 44 measurements). These findings support our hypothesis that steroid treatment results in thinning of the ileal submucosa in the newborn period.

IGF-I immunolocalization in the submucosa of the ileum positively correlates with IGF-I genotype. Immunolocalization of IGF-I in fixed, frozen sections revealed three distinctly different patterns of localization between heterozygous IGF-I knockout, wild type and homozygous IGF-I over-expresser mice. Heterozygous IGF-I knockouts showed little or no IGF-I localization in any of the tissues associated with the bowel wall (Fig. $4 A$ ), which was comparable to IGF-I localization seen in
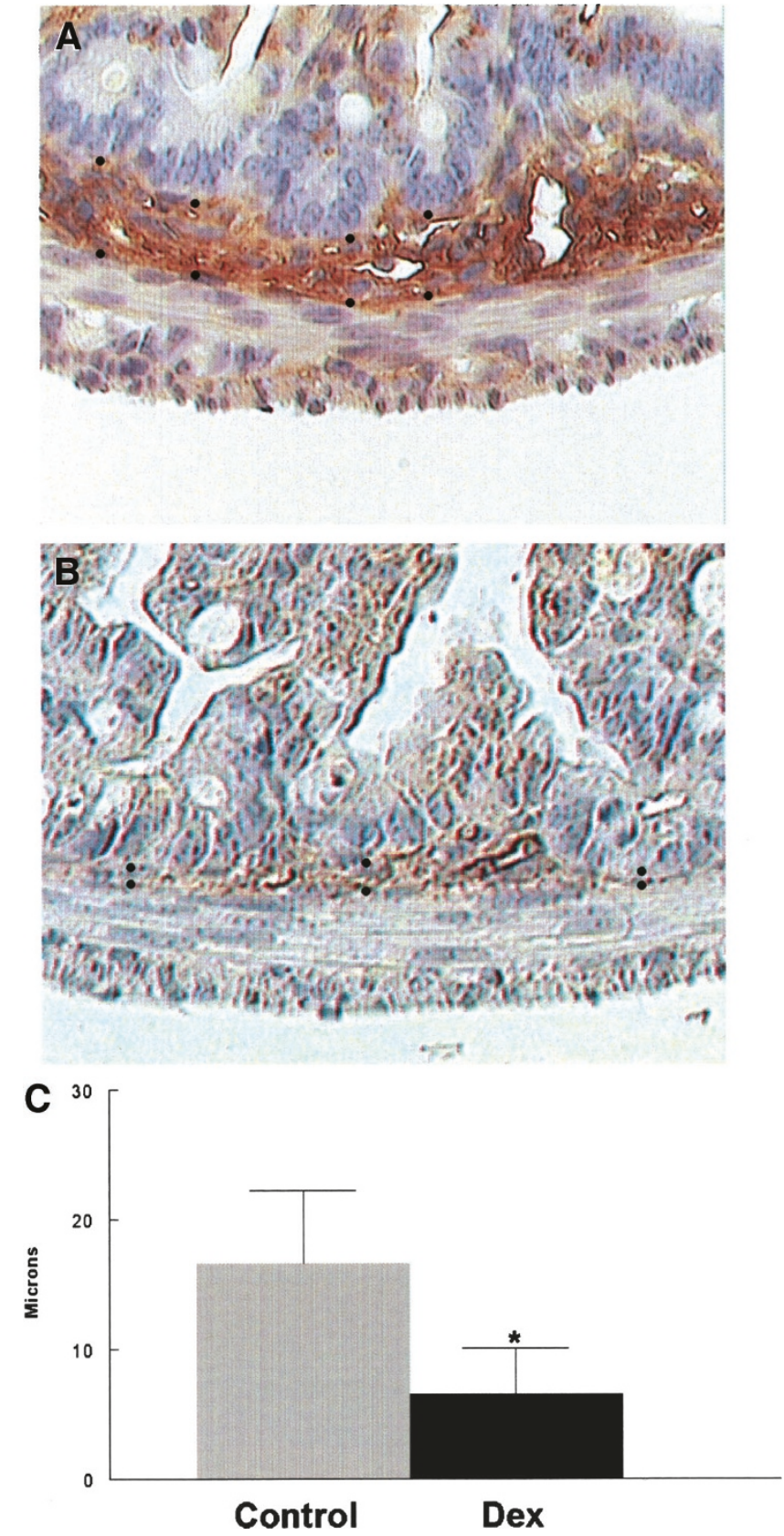

Figure 3. Dexamethasone treatment reduces IGF-I and significantly thins the submucosa. (A) Representative IGF-I immunolocalization in an ileal cross section from a control mouse. IGF-I staining (in brown) is abundant in the submucosa. ( $B$ ) Representative IGF-I immunolocalization in an ileal cross section of a dexamethasone-treated mouse. IGF-I abundance (in brown) is diminished compared with control. Black dots indicate submucosal boundaries at measurement points. $(C)$ Mean submucosal thickness $(\mu \mathrm{m})$ is displayed with $\mathrm{SD}$ for control and dexamethasone-treated mouse ileum $(* p<0.01)$.

dexamethasone-treated animals (Fig. 3B). Wild types showed submucosal staining similar to that seen in mice that received intraperitoneal injections of saline vehicle (Fig. $4 B$ versus Fig. $3 A$ ), such that staining was intense within the submucosa but minimal or absent in surrounding tissue layers. Homozygous IGF-I over-expressers showed increased submucosal staining in comparison to wild types, although this increase appeared to be more secondary to an increase in submucosal thickness rather than an increase in staining intensity (Fig. 4C). 

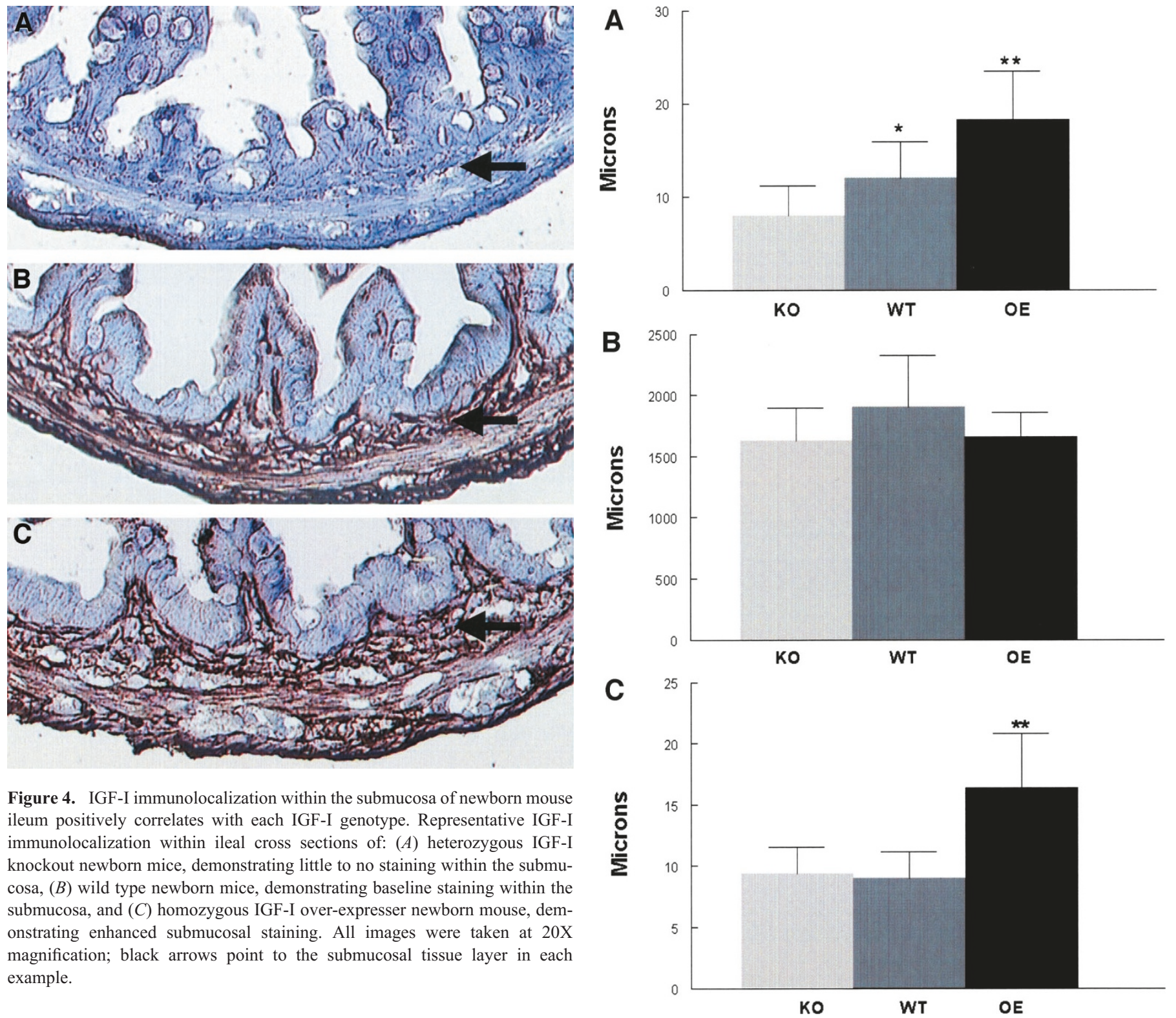

Figure 4. IGF-I immunolocalization within the submucosa of newborn mouse ileum positively correlates with each IGF-I genotype. Representative IGF-I immunolocalization within ileal cross sections of: $(A)$ heterozygous IGF-I knockout newborn mice, demonstrating little to no staining within the submucosa, $(B)$ wild type newborn mice, demonstrating baseline staining within the submucosa, and $(C)$ homozygous IGF-I over-expresser newborn mouse, demonstrating enhanced submucosal staining. All images were taken at 20X magnification; black arrows point to the submucosal tissue layer in each example.

Submucosal thickness in the ileum of newborn mice positively correlates with each IGF-I genotype. Measurements of submucosal thickness showed significant differences between each of the IGF-I genotypes $(p<0.05)$. The mean submucosal thickness with standard deviations for knockouts $(n=4$ mice and 15 measurements) was $7.98 \pm 3.16 \mu \mathrm{m}$, wild types $(n=$ 4 mice and 17 measurements) was $12.00 \pm 3.89 \mu \mathrm{m}$ and over-expressers ( $n=4$ and 18 measurements) was $18.20 \pm$ $5.25 \mu \mathrm{m}$. Heterozygous IGF-I knockout mice had significantly thinner submucosa when compared with wild types, whereas homozygous IGF-I over-expressers were significantly thicker when compared with wild types (Fig. 5A). Because we have previously shown that ileal circumference alters the thickness of bowel wall tissues (9), we also measured the circumference from these same tissue sections to investigate this possibility between the three IGF-I genotypes. The mean ileal circumference for knockouts was $1660.24 \pm 193.88 \mu \mathrm{m}$, wild types was $1904 \pm 419.35 \mu \mathrm{m}$ and over-expressers was $1627.52 \pm 268.64$ $\mu \mathrm{m}$. No significant difference was seen between the three conditions (Fig. 5B). Taken together, these findings suggest

Figure 5. Submucosal thickness positively correlates with each IGF-I genotype. $(A)$ Submucosal thickness $(\mu \mathrm{m})$ is significantly thinner in IGF-I heterozygous knockouts (KO) when compared with wild types (WT) and is significantly thicker in IGF-I homozygous over-expressers (OE) when compared with WT. $(B)$ Ileal lumen circumference is not significantly different between the three genotypes. $(C)$ Muscularis thickness $(\mu \mathrm{m})$ is not significantly different between KO and WT but is significantly thicker in OE compared with WT. For each figure: $*=p<0.05$ versus WT; $* *=p<0.05$ versus both $\mathrm{KO}$ and $\mathrm{WT}$; error bars $=\mathrm{SD}$; and $n=4$ mice for each of the three genotypes.

that increasing IGF-I genotype (and the resulting IGF-I abundance within the submucosa) governs submucosal growth and thickness in the newborn mouse ileum.

Muscularis thickness in the ileum of newborn mice is increased in homozygous IGF-I over-expressers. Measurements of muscularis thickness showed a varied response to IGF-I genotype. Heterozygous IGF-I knockout mice did not have a significant difference from that of wild types (9.39 \pm $2.16 \mu \mathrm{m}$ versus $8.96 \pm 2.21 \mu \mathrm{m}$ respectively). However, homozygous IGF-I over-expressers had a significantly thicker muscularis when compared with wild types $(8.96 \pm 2.21 \mu \mathrm{m}$ 
versus $16.41 \pm 4.39 \mu \mathrm{m}$, respectively) (Fig. 5C). These findings suggest that smooth muscle growth within the neonatal ileum can be augmented by IGF-I but that IGF-I may be less crucial for maintenance of smooth muscle mass during this period of development.

\section{DISCUSSION}

Our findings in this study support the hypothesis that dexamethasone-induced thinning of the bowel wall is secondary to diminished IGF-I abundance within the submucosa of the neonatal ileum. Both heterozygous IGF-I knockout and homozygous IGF-I over-expresser newborn mice demonstrate altered submucosal IGF-I abundance and growth (measured by thickness) at $3 \mathrm{~d}$ of life. Within this time period, IGF-I paucity in the submucosa correlated with a thinner submucosa (both in the heterozygous IGF-I knockouts and in dexamethasonetreated animals), whereas IGF-I over-expression resulted in a thicker submucosa. This relatively short window of development may have profound relevance to the structural integrity of the ileal bowel wall, since the submucosal tissue layer has the greatest tensile strength.

Because the submucosa is highly vascularized and is presumably in equilibrium with total body serum, it could be that systemic changes in IGF-I availability (as seen during comparisons between IGF-I knockout and IGF-I over-expresser mice) drive the observed changes in IGF-I abundance within the submucosa (16). Glucocorticoids are known to reduce serum concentrations of IGF-I in human neonates and we have shown in previous work that they reduce submucosal IGF-I immunolocalization in newborn mice $(9,16)$. In this study, we also demonstrate that dexamethasone treatment significantly thins the submucosa. Taken together, these findings suggest that submucosal IGF-I abundance is proportional to the total serum IGF-I concentration and that both measures are responsive to glucocorticoids during this period of development.

Diminished IGF-I abundance does not appear to cause ileal muscularis thinning during the first three days of life. We have previously demonstrated that ileal muscularis thickness is inversely proportional to lumen diameter (9). In this study, we found no thinning of the muscularis in heterozygous IGF-I knockout mice (which had comparable lumen diameters to that of wild type mice). In contrast, we did see significant thickening of the muscularis in homozygous IGF-I over-expresser mice. These findings suggest that gut smooth muscle myocytes are responsive to IGF-I (a phenomenon well documented in cell culture) $(17,18)$ but are less dependent upon IGF-I for baseline growth in vivo during the neonatal period.

Our data confirm that IGF-I governs ileal submucosal growth and thickness in the neonatal period and that this specific aspect of gut development is dramatically perturbed by exogenous glucocorticoids. These findings have direct rele- vance to an emerging neonatal disease known as spontaneous intestinal perforation and demonstrate a mechanism by which glucocorticoids likely perturb ileal development in the extremely low birth weight infant. Perhaps more important, the newborn mouse model is proving to be a valuable model for investigating the interplay between the adrenal-corticosteroid axis and tissue-specific effects of growth factors $(9,19)$. Understanding this endocrine-to-paracrine balance will be essential as we confront the medical challenges associated with increasing gastrointestinal immaturity in the neonate.

Acknowledgments. The authors thank Sarabeth L. Thomas M.S., for proof reading and helpful discussions.

\section{REFERENCES}

1. Gordon PV, Young ML, Marshall DD 2001 Focal small bowel perforation: an adverse effect of early postnatal dexamethasone therapy in extremely low birth weight infants. J Perinatol 21:156-160

2. Gordon P, Rutledge J, Sawin R, Thomas S, Woodrum D 1999 Early postnatal dexamethasone increases the risk of focal small bowel perforation in extremely low birth weight infants. J Perinatol 19:573-577

3. Garland JS, Alex CP, Pauly TH, Whitehead VL, Brand J, Winston JF, Samuels DP, McAuliffe TL 1999 A three-day course of dexamethasone therapy to prevent chronic lung disease in ventilated neonates: a randomized trial. Pediatrics 104:91-99

4. Stark AR, Carlo WA, Tyson JE, Papile LA, Wright LL, Shankaran S, Donovan EF, Oh W, Bauer CR, Saha S, Poole WK, Stoll BJ 2001 Adverse effects of early dexamethasone in extremely-low-birth-weight infants. National Institute of Child Health and Human Development Neonatal Research Network. N Engl J Med 344:95-101

5. Nanthakumar NN, Henning SJ 1995 Distinguishing normal and glucocorticoid-induced maturation of intestine using bromodeoxyuridine. Am J Physiol 268:G139-G145

6. Henning S, Rubin D, Shulman R 1994 Ontogeny of the intestinal mucosa. In: Johnson L, editor. Physiology of the gastrointestinal tract. Raven, New York, pp 571-610

7. Burrin DG, Wester TJ, Davis TA, Fiorotto ML, Chang X 1999 Dexamethasone inhibits small intestinal growth via increased protein catabolism in neonatal pigs. Am J Physiol 276:E269-E277

8. Tatekawa Y, Muraji T, Imai Y, Nishijima E, Tsugawa C 1999 The mechanism of focal intestinal perforations in neonates with low birth weight. Pediatr Surg Int 15:549-552

9. Gordon PV, Price WA, Stiles AD 2001 Dexamethasone administration to newborn mice alters mucosal and muscular morphology in the ileum and modulates IGF-I localization. Pediatr Res 49:93-100

10. Liu JP, Baker J, Perkins AS, Robertson EJ, Efstratiadis A 1993 Mice carrying null mutations of the genes encoding insulin-like growth factor I (Igf-1) and type 1 IGF receptor (Igf1r). Cell 75:59-72

11. Ohneda K, Ulshen MH, Fuller CR, D’Ercole AJ, Lund PK 1997 Enhanced growth of small bowel in transgenic mice expressing human insulin-like growth factor I. Gastroenterology 112:444-454

12. D'Ercole AJ 1993 Expression of insulin-like growth factor-I in transgenic mice. Ann NY Acad Sci 692:149-160

13. Pete G, Fuller CR, Oldham JM, Smith DR, D'Ercole AJ, Kahn CR, Lund PK 1999 Postnatal growth responses to insulin-like growth factor I in insulin receptor substrate-1-deficient mice. Endocrinology 140:5478-5487

14. Laemmli UK 1970 Cleavage of structural proteins during the assembly of the head of bacteriophage T4. Nature 227:680-685

15. Keller TC, III, Gordon PV 1991 Discrete subcellular localization of a cytoplasmic and a mitochondrial isozyme of creatine kinase in intestinal epithelial cells. Cell Motil Cytoskeleton 19:169-179

16. Bloomfield FH, Knight DB, Breier BH, Harding JE 2001 Growth restriction in dexamethasone-treated preterm infants may be mediated by reduced IGF-I and IGFBP-3 plasma concentrations. Clin Endocrinol (Oxf) 54:235-242

17. Kuemmerle JF 1997 Autocrine regulation of growth in cultured human intestinal muscle by growth factors. Gastroenterology 113:817-824

18. Kuemmerle JF, Bushman TL 1998 IGF-I stimulates intestinal muscle cell growth by activating distinct PI 3-kinase and MAP kinase pathways. Am J Physiol 275:G151G158

19. Gordon PV, Price WA, Stiles AD, Rutledge JC 2001 Early postnatal dexamethasone diminishes transforming growth factor alpha localization within the ileal muscularis propria of newborn mice and extremely low-birth-weight infants. Pediatr Dev Pathol $4: 532-537$ 